\title{
Discrimination of black onion flavor using the electronic nose
}

\author{
Dan WANG ${ }^{1, a}$, Panpan LIU ${ }^{1,2, c}$, Yue MA ${ }^{1, b}$, Yubin Wang ${ }^{1, c}$ and Xiaoyan \\ $\mathrm{ZHAO}{ }^{1, \mathrm{c}, *}$
}

${ }^{1}$ China;Beijing Vegetable Research Center, Beijing Academy of Agriculture and Forestry Science, Key Laboratory of Biology and Genetic Improvement of Horticultural Crops (North China), Ministry of Agriculture, P.R.China, Key Laboratory of Urban Agriculture (North), Ministry of Agriculture, Beiji ng Key Laboratory of agricultural products of fruits and vegetables preservation and processing,

\author{
P.R. Beijing, 100097, China; \\ ${ }^{2}$ Food Institute ,Shihezi University, Xinjiang, 833200, \\ awangdan@nercv.org, ${ }^{b}$ mayue@nercv.org, 'chaoxiaoyan@nercv.org
}

Keywords: black onion, flavor, electronic nose.

Abstract: The flavor of onions at different processing times (0, 6, 1420 days) were monitored by electronic nose. Different sensors were sensitive to different compounds. The results showed that the sulfur compounds were the major components in the onions, which decreased significantly with the increasing of the processing time. What is more, the flavor of the onions could be differentiated clearly by electronic nose. The linear discriminant analysis (LDA) model could be applied to distinguish the samples efficiently.

\section{Introduction}

Onion is one of the most important spices in china and other countries, such as Indian and America. It is famous for its unique flavor and functional properties. There have been many researchers reported that onion was a major source of antioxidants. Bora Lee had reported that onion could enhance the activity of plasma SOD and GPx and inhibit the liver lipid peroxidation [1]. Besides, because of the higher content of organosulfur compounds [2], it had widely used as feed additives [3], food ingredients [4] and anti-infammatory [5]. Today, onion was consumed as fresh, as oil, and in paste in the market currently. In order to change the eating quality, fermention was used to process the product. Black onion was a rising functional foods that made from raw onion in hot and humid environment for nearly one month.

Electric nose is an intelligent technique that simulated the biological sense of smell. It had been used to investigate and control quality of food and vegetables [6], monitor the shelf life of the product [7], and distinguish the flavor characteristics [8].

The aim of this study was to monitor the change in flavor of the black onion during the processing using PEN 2 E-nose by PCA.

\section{Materials and methods}

\section{Materials}

Purple onion was obtained from a local market in Beijing.

\section{Experimental setup}

The whole purple onion was placed in a humidity Chamber (GDS-225, Yashilin, Beijing), which procedure set at $70{ }^{\circ} \mathrm{C}$ under $90 \%$ relative humidity ( $\left.\mathrm{RH}\right)$ for 20 days. The onion was removed out under different times $\left(0,6,14,20\right.$ days). All the samples were maintained at $-80{ }^{\circ} \mathrm{C}$ until analysis. 


\section{Electric nose measurement}

All the experiments were performed using an AIRSENSE PEN3 electronic nose, which consist of 10 different metal oxide sensors. The different sensors were sensitive to different areas [9]. The instruments should be pre-warmed for $30 \mathrm{~min}$ prior the measurement. $2 \mathrm{~g}$ onion paste was weighted and placed in a sealed vial with volume of $20 \mathrm{~mL}$. Before analysis, the system should be purged with the ambient air under 180 s for 2 times. Then, the samples were conducted for 60 s for analyzing. It should be noted that the system must be cleaned for 180s again before the next sample was inlet.

\section{Data collection and statistical analyses}

The data between 48s-52s were utilized. Principal component analysis (PCA) and linear discriminant analysis (LDA) were performed by WinMuster software to discriminate between the different classes of samples. All samples were repeated in three times.

\section{Results and Discussion}

\section{The sensor of onion aroma}

Fig. 1 shows a typical signal of 10 sensors for onion which at different times. Each curve represents one sensor response value. The G/G0 (G0/G) values response to volatiles were varied with different samples. As for onion, it can be seen that sensor 2, 7 and 9 were increased remarkable. The rest sensors almost had not change. Sensor 2, 7 and 9 were sensitive to nitrogen oxides, sulfur compounds and aromatics compounds, sulfur organic compounds, respectively. As is known that, onion is rich in sulfur compounds, which illustrated that the electronic nose could explain the flavor of the onion. Fig. 2 displays the response values of electronic nose sensors for onion at $0,6,14,20$ days. As shown in the figure, the values of the three important sensors decreased significantly with the increasing of the time. It is consistent with the human sensory, which found that the pungent flavor of onion at $20^{\text {th }}$ days decreased significantly. The taste of the onion became more easily acceptable by people.

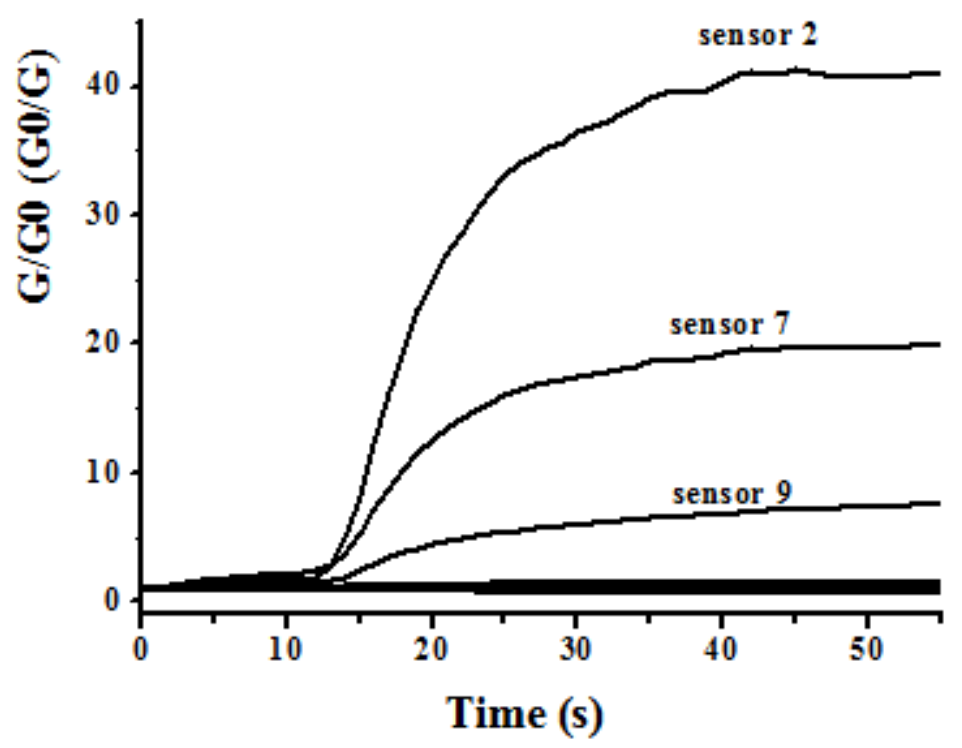

Fig. 1 The graph for response of sensors to onion aroma 


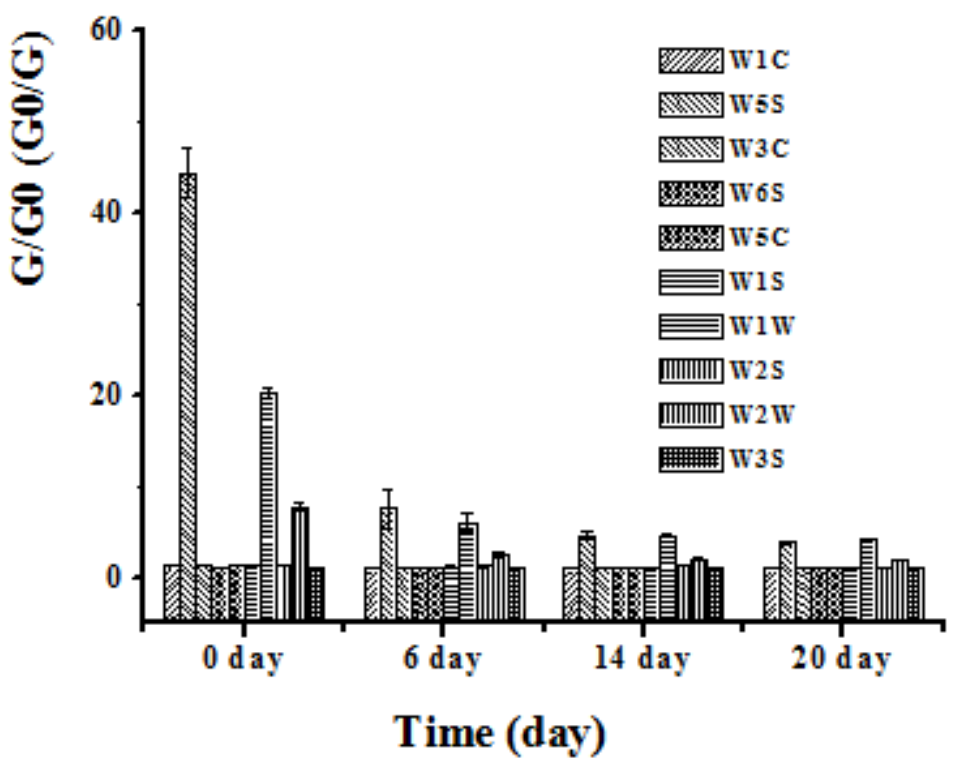

Fig. 2 Response values of electronic nose sensors for different onions

\section{Principal component analysis (PCA) and linear discriminant analysis (LDA)}

The PCA result was shown in Fig. 3. The PCA shows the differentiation of the four kinds of onions. According to the PCA analysis, two main principal components were obtained for the onions, which PC1 explained $99.97 \%$ of the total data variance, PC2 explained $0.03 \%$. The onion at 0 day could be distinguished clearly from others, whereas the differentiation between the onions at 6,14, 20 days were not so clear. Linear discriminant analysis (LDA) was a useful method to maximize the differentiation between the samples. As shown in LDA plot, two liner discriminants explained $95.99 \%$ of the total variance (LD1 for $81.51 \%$ and LD2 for $14.48 \%)$ ). From the plot, it can be seen that he electronic nose was able to differentiate clearly the onions at different periods $(0,6,14,20$ days).

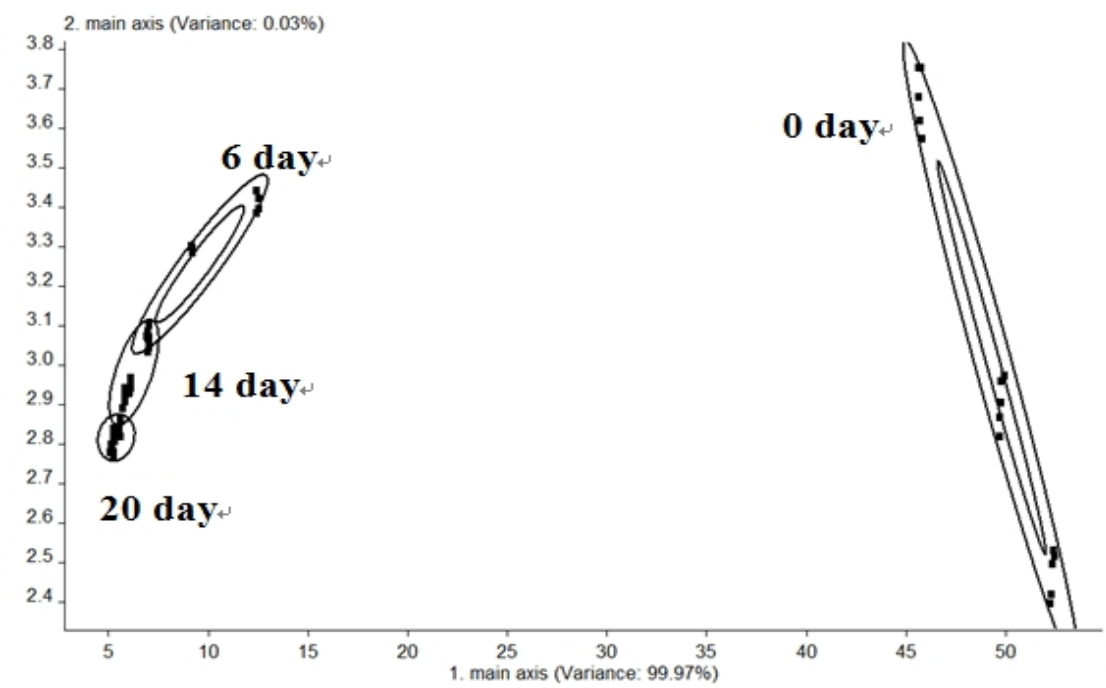

Fig. 3 PCA analysis for the onion processed for different times 


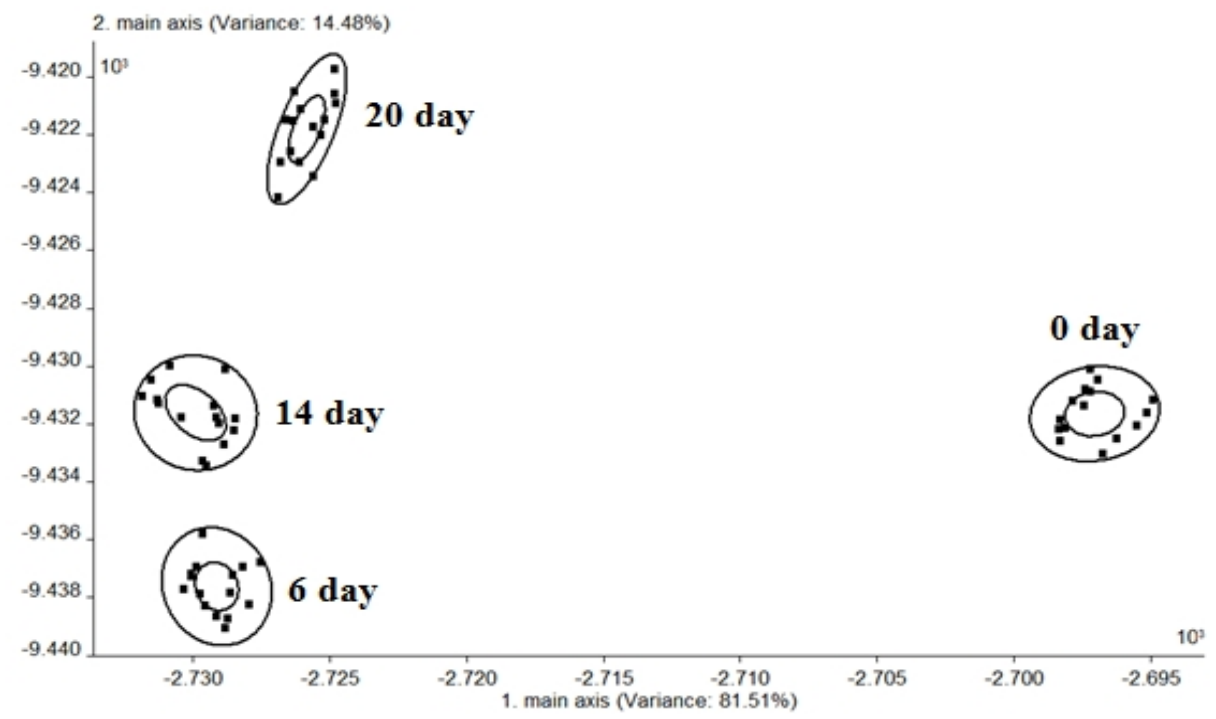

Fig. 4 LDA analysis for the onion processed for different times

\section{Conclusions}

The results of this study explained that electronic nose could be applied to distinguish the odor of onions at different times efficiently. What is more, LDA model could differentiate the samples more clearly comparing to the PCA model.

\section{Acknowledgements}

This work was supported by China Agriculture Research System (CARS-25) and Foundation of Beijing Key Laboratory of fruits and vegetables preservation and processing (Z141105004414037).

\section{References}

[1] Lee, B., Jung, J.H., Kim, H.S., 2012. Assessment of red onion on antioxidant activity in rat. Food and Chemical Toxicology 50, 3912-3919.

[2] Lanzotti, V., 2006. The analysis of onion and garlic. Journal of Chromatography A 1112, 3-22.

[3] Goodarzi, M., Nanekarani, G., 2014. Effect of onion extract in drink water on performance and carcass traits in broiler chickens. IERI Procedia 8, $107-112$.

[4] Eduvigis Roldán, Concepción Sánchez-Moreno, et al, 2008. Characterisation of onion (Allium cepa L.) by-products as food ingredients with antioxidant and antibrowning properties. Food Chemistry 108, 907-916.

[5] Natasa, S., Dejan, O., Dragana, C.S., et al, 2013. Phenolic profile, antioxidant, anti-inflammatory and cytotoxic activities of small yellow onion (Allium flavum L. subsp. flavum, Alliaceae). LWT - Food Science and Technology 54, 139-146.

[6] Abbott, J.A., 1999. Quality measurement of fruits and vegetables. Postharvest Biology and Technology $15,207-225$.

[7] Antihus Hernández Gómez, Wang, J., et al, 2008. Monitoring storage shelf life of tomato using electronic nose technique. Journal of Food Engineering 85, 625-631.

[8] Grigioni , G., Carduza, F., et al, 2004. Flavour characteristics of Ilex paraguariensis infusion, a typical Argentine product, assessed by sensory evaluation and electronic nose. J Sci Food Agric 84, 427-432.

[9] Liu, P., Tu, K., 2012. Prediction of TVB-N content in eggs based on electronic nose. Food Control 23, $177 \mathrm{e} 183$. 